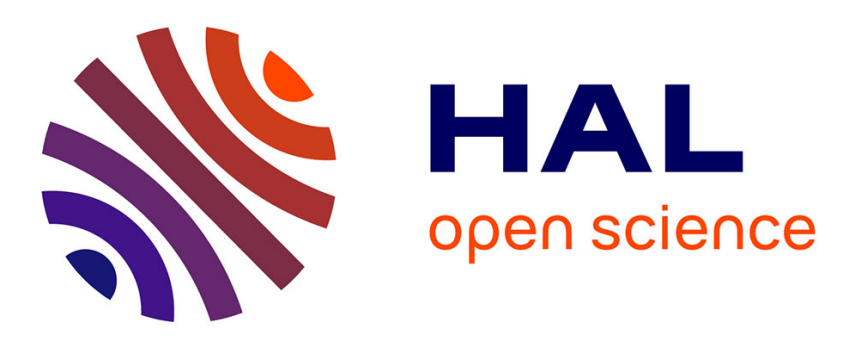

\title{
Validation of structural dynamics models containing uncertainties
}

Pierre Ladevèze, Guillaume Puel, Arnaud Deraemaeker, Thierry Romeuf

\section{To cite this version:}

Pierre Ladevèze, Guillaume Puel, Arnaud Deraemaeker, Thierry Romeuf. Validation of structural dynamics models containing uncertainties. Computer Methods in Applied Mechanics and Engineering, 2006, 195 (4-6), pp.373-393. 10.1016/j.cma.2004.10.011 . hal-00258640

\section{HAL Id: hal-00258640 \\ https://hal.science/hal-00258640}

Submitted on 22 Feb 2008

HAL is a multi-disciplinary open access archive for the deposit and dissemination of scientific research documents, whether they are published or not. The documents may come from teaching and research institutions in France or abroad, or from public or private research centers.
L'archive ouverte pluridisciplinaire HAL, est destinée au dépôt et à la diffusion de documents scientifiques de niveau recherche, publiés ou non, émanant des établissements d'enseignement et de recherche français ou étrangers, des laboratoires publics ou privés. 


\title{
Validation of structural dynamics models containing uncertainties
}

\author{
P. Ladevèze ${ }^{\mathrm{a}, *}$, G. Puel $^{\mathrm{a}}$, A. Deraemaeker ${ }^{\mathrm{a}, 1}, \mathrm{~T}$. Romeuf ${ }^{\mathrm{b}}$ \\ ${ }^{a}$ LMT-Cachan (ENS Cachan/CNRS/Paris 6 University) \\ 61 avenue du Président Wilson 94235 Cachan Cedex France \\ ${ }^{\mathrm{b}}$ European Aeronautic Defence and Space Company (EADS Space Transportation) \\ 66 route de Verneuil BP 3002 78133 Les Mureaux Cedex France
}

\begin{abstract}
This paper deals with model validation in structural dynamics for a family of quasiidentical structures in the context of uncertain measurements. The crucial point is for the engineer to be able to quantify the quality of the model, which is probabilistic with respect to a set of measurements from which a probability density function can be extracted. Our approach is based on the "mechanical concept" of Constitutive Relation Error Estimator (CRE), which was introduced initially in order to quantify the quality of finite element analyses, then developed in the deterministic context. Our extended CRE estimator enables us to quantify the quality of a given probabilistic model and, thus, to update and validate the model. Several examples are given, including an industrial case.
\end{abstract}

Key words: validation, dynamics, modeling, uncertainties

* Corresponding author.

Email addresses: pierre.ladeveze@lmt.ens-cachan.fr (P. Ladevèze), guillaume.puel@lmt.ens-cachan.fr (G. Puel), arnaud.deraemaeker@ulb.ac.be (A. Deraemaeker), thierry.romeuf @space.eads.net (T. Romeuf).

1 Present address: ULB-Active Structures Laboratory, 50 avenue F. Roosevelt CP 165/42, 1050 Brussels Belgium 


\section{Introduction}

Modeling tools constitute a major accomplishment of the past 25 years, if one considers how radically they transformed, and continue to transform, industry and research. This is a true revolution which is far from complete. Of course, mastering the models (by which we mean controlling the calculation process itself as well as validating the results against a set of experimental data) is an essential requirement in using these tools. Until now, model validation and verification have been carried out mostly in a deterministic sense. Indeed, for deterministic models, there are a number of open issues which still require research, but one must recognize that most of the scientific obstacles have been eliminated [1-3]. On the contrary, regarding the stochastic domain, one could jokingly say that practically everything remains to be done. One should also add that the ability to deal with uncertain data in the model's parameters and in the environment is fast becoming a priority in structural analysis, particularly in the industrial context.

This work focuses on validation alone; more precisely, its purpose is to derive tools enabling one to master stochastic models in a possibly uncertain environment. Our approach is an extension of the constitutive relation error method, which is extremely effective in the deterministic case [4].

Validation (in fact, model updating) has been widely studied, particularly for the deterministic case and for structural dynamic models, as shown by stateof-the-art reviews in $[5,6]$. Many of the methods proposed did not attempt to provide a meaningful error measure which could be used for validation. The earlier model updating methods fall into the category of 'direct methods', in which corrections to the mass and stiffness matrices of the model are sought without taking into account the physical meaning of the modifications. Within this category, a first set of methods is based on the search for minimum norm corrections $[7,8]$. A second set of methods is closely related to control theory $[9,10]$. The main drawback of these methods is that the corrections usually lack physical meaning; consequently, the models could be invalid when applied to configurations other than those used for the updating process.

In order to preserve the physical meaning of the model, indirect or parametric methods have been developed. In these methods, the changes in the stiffness and mass matrices are based on variations of the physical parameters of the model. The approach consists in building a cost function which represents the correlation between the numerical model and the test data in terms of the physical parameters of the model. Several types of cost functions can be used, such as input residuals $[11,12]$ and output residuals $[13,14]$.

As mentioned earlier, many of these methods do not attempt to perform quan- 
titative validation. This field is still quite new; some attempts for nonlinear models can be found in [15-17].

At Cachan, still for the deterministic case, we have been developing a rather different approach which has a strong mechanical meaning. This approach can be viewed as an extension of works previously conducted on a posteriori estimators in order to quantify the quality of a finite element calculation, e.g. in studying the situation where the approximate model is the numerical model $[2,1]$. Our method is based on the concept of a posteriori Constitutive Relation Error (CRE), which is a meaningful energy-based indicator. Using this concept, we are able to address the validation question as well as update the model. The initial works on model updating go back to the eighties [18]. The first development of the method was aimed at updating the model based on eigenfrequencies and eigenmodes [19]. Then, the method was extended to forced vibration problems in [20]. This approach is based on what is known as the Drucker error, and it has proved its effectiveness in updating the mass, stiffness and damping properties [21,22]. The method can also deal with nonlinearities due to the material's behavior or to contacts. The concept of dissipation error [23] was introduced in a further development presented in [24]. This error has a clear mechanical meaning and emphasizes the dissipation properties of the model. Let us also mention the existence of very similar methods for free vibration problems, such as the Minimum Dynamic Residual Expansion (MDRE) method [25] or the Modeling Error in the Constitutive Equations (MECE) method [26,27].

In order to get closer to reality, uncertainties must be introduced into the model's parameters (material characteristics, parameters of the joints), leading to a probabilistic structural model. Regarding experimental data, which are not necessarily the ultimate reference in the case of industrial structures, measurement errors must be taken into account. In industrial tests, in addition to noise due to the testing equipment, significant measurement errors (such as wiring errors, erroneous excitation direction, errors in the test reports) often occur and cause the updating methods to fail; in order to deal with these problems, a method for recovering consistent experimental data was proposed recently [28]. Consequently, the actual validation problem concerns the combination of a given probabilistic model with noisy measurements.

In the stochastic case, major progress has been made recently in structural analysis, particularly with the Karhunen-Loeve method and the polynomial chaos technique, which can be found in [29-31]. Conversely, as mentioned above, very little research has been done on the verification and validation of probabilistic models. Nevertheless, one should mention, in relation to verification/validation, the error indicators proposed in [32,33], which are, in fact, sensitivity indicators and, therefore, have nothing in common with what has been developed in the deterministic case. Regarding validation, one should 
mention what is known as "inverse methods", many of which definitely fall into the stochastic category [34,35]; in fact, in these methods, the approach is purely stochastic and mechanics is mostly absent; a first attempt to draw a parallel between the $\mathrm{CRE}$ and the general inverse problem theory can be found in [36], where it is proposed to use the local value of the CRE to evaluate the relative variances on the parameters of the model. In [37], a statistical interpretation of the norm to be applied to the measurements in direct relation to the covariance matrix was advocated as being the only possible choice if the data are scarce for a first true attempt to extend the constitutive relation error to the stochastic case. In [38], this statement was shown to be excessive and equally effective choices were presented. In this paper, we go further than these previous works, considering that the model is probabilistic, too.

The approach we are following here is radically different from any existing work in the stochastic case. It is deliberately "mechanics-oriented" and derives from works on verification and validation we did for the deterministic case based on the concept of constitutive relation error. The extension of the constitutive relation error to the stochastic case presents no serious difficulty in the particular case where the model is deterministic and the experimental data contain noise $[4,38]$. Through a number of examples, it was shown that the approach, which is purely mechanical, is perfectly consistent.

Here, new illustrations are given. The main difficulty (in fact, the real scientific stumbling block) lies in the validation of a stochastic model. The problem consists in deriving an error measure which is zero if the model is "exact", i.e. if the results obtained from the model match the experimental data. The extension to the modified constitutive relation error discussed here satisfies this fundamental requirement: it is based on [38]. It is still completely "mechanicsoriented" regarding the norms used. Another constraint is that the statistical description of the experimental data is often very limited. In this regard, a major feature of our approach is that we propose to reconstruct these experimental data using the statistical mean value and the model; this reconstruction is based on a qualitative analysis of the error in the response in terms of the two sources of errors, i.e. the fluctuations of the parameters and the possible variations due to the approximate nature of the stochastic model itself. The "excitation" can be deterministic or not. In this paper, the simplest possible "model" problem, i.e. an elastic structure with small perturbations in isothermal conditions, is described in full detail. The extension to the nonlinear case, whether quasi-static or dynamic, presents no major a priori difficulty. Finally, the approach is illustrated by numerical examples. 


\section{The "model" reference problem}

\subsection{Description of the problem}

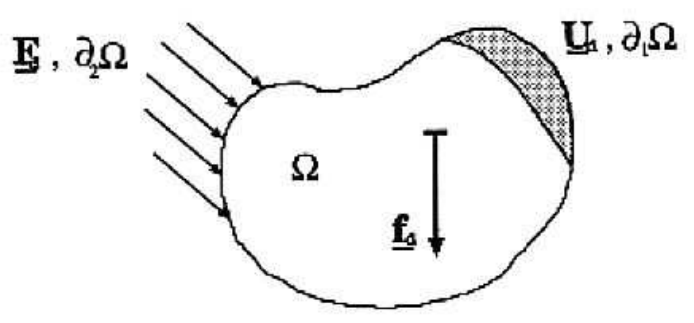

Fig. 1. Schematic description of the problem

Here, we are considering the simplest problem possible, which is the elastic modeling of a structure under quasi-static conditions with small perturbations. The model, however, is probabilistic. The Hooke's tensor and the data are stochastic fields. Nevertheless, through the Karhunen-Loeve method, it is usual and convenient to reduce these to stochastic variables [29-31]. It is also possible and easy to preserve the positive definite character of the Hooke's operator [30]. The study of the underlying approximations is not within the scope of this work. Then, the reference "model" problem becomes:

Find $\underline{U}(\underline{M}, \theta), \underline{\sigma}(\underline{M}, \theta), \underline{M} \in \Omega$ such that:

- kinematic constraint equations $\underline{U} \in \mathcal{U}$

$$
\underline{U}_{\mid \partial_{1} \Omega}=\underline{U}_{\mathrm{d}}(\underline{d}(\theta))
$$

- equilibrium equations $\sigma \in \mathcal{S}$

$$
\begin{gathered}
\underline{\operatorname{div}} \sigma+\underline{f}_{\mathrm{d}}(\underline{d}(\theta))=\underline{0} \\
\sigma \underline{n}_{\partial_{2} \Omega}=\underline{F}_{\mathrm{d}}(\underline{d}(\theta))
\end{gathered}
$$

- constitutive relation

$$
\sigma=\mathbf{K}(\underline{m}(\theta)) \varepsilon(\underline{U})
$$

The Hooke's operator is a function of the vector random variable $\underline{m}(\theta)$, whose probability density function and corresponding space are $\mathrm{d} P(\underline{m})$ and $\mathbf{H}_{\mathrm{m}}$ respectively. The quantities $\underline{U}_{\mathrm{d}}, \underline{f}_{\mathrm{d}}, \underline{F}_{\mathrm{d}}$ are functions of the vector random variable $\underline{d}(\theta)$, whose probability density function and corresponding space are $\mathrm{d} Q(\underline{d})$ and $\mathbf{H}_{\mathrm{d}}$ respectively. All these quantities are assumed to be perfectly known; they characterize the model we are seeking to validate. 
In fact, the solution $s(\underline{M}, \theta)=(\underline{U}(\underline{M}, \theta), \underline{\sigma}(\underline{M}, \theta))$ can be written as:

$$
s(\underline{M}, \underline{m}(\theta), \underline{d}(\theta))
$$

A major point is that $s(\underline{M}, \underline{m}, \underline{d})$ is the solution of a parameterized deterministic problem whose parameters are $\underline{m}$ and $\underline{d}$. The resolution of such a problem presents no real difficulty, except in the nonlinear domain or if the number of parameters is really very large. We will not concern ourselves with these cases; we will consider the situation where the problem can be properly solved using, for example, the "polynomial chaos" technique coupled with a finite element approach [29,31].

\subsection{Extension of the CRE to the stochastic framework}

Definition: $\hat{s}(\underline{m}, \underline{d})$ is admissible if $\forall \underline{d} \in \mathbf{H}_{\mathrm{d}}$ it verifies:

- the kinematic constraint equations

- the equilibrium equations

The corresponding space is $\mathbf{S}_{\text {ad }}^{\mathrm{d}}$. The only equation which is not verified by $\hat{s}$ is the constitutive relation, whose residual at any point $\underline{M}$ of domain $\Omega$ is:

$$
e_{C R E}=\hat{\sigma}(\underline{m}, \underline{d})-\mathbf{K}(\underline{m}) \varepsilon(\underline{\hat{U}}(\underline{m}, \underline{d}))
$$

It is also useful to define the global constitutive relation error:

$$
\begin{gathered}
\bar{E}_{C R E}^{2}=\frac{\int_{\mathbf{H}_{\mathrm{m}} \times \mathbf{H}_{\mathrm{d}}} E_{C R E}^{2}(\underline{m}, \underline{d}) \mathrm{d} P(\underline{m}) \mathrm{d} Q(\underline{d})}{\bar{D}^{2}} \\
\bar{D}^{2}=\int_{\mathbf{H}_{\mathrm{m}} \times \mathbf{H}_{\mathrm{d}}} \int_{\Omega}\left[\varphi(\hat{\varepsilon})+\varphi^{*}(\hat{\tilde{\sigma}})\right] \mathrm{d} \Omega \mathrm{d} P(\underline{m}) \mathrm{d} Q(\underline{d}) \\
E_{C R E}^{2}=\int_{\Omega}\left[\varphi(\hat{\varepsilon})+\varphi^{*}(\hat{\sigma})-\operatorname{Tr}[\hat{\sigma} \hat{\varepsilon}]\right] \mathrm{d} \Omega
\end{gathered}
$$

where $\varphi, \varphi^{*}$ are the classical potentials for the constitutive relation in elasticity.

Besides, one can easily show that:

Property 1: The global constitutive relation error is zero if and only if $\hat{s}(\underline{m}, \underline{d})$ is the exact solution of the reference problem.

Property 2: If $s_{e x}=\left(\underline{U}_{e x}, \sigma_{e x}\right)$ is the exact solution, one has:

$$
\left\|\hat{\sigma}-\sigma_{e x}\right\|_{\mathcal{S}}^{2}+\left\|\underline{\hat{U}}-\underline{U}_{e x}\right\|_{\mathcal{U}}^{2}=\bar{E}_{C R E}^{2} \bar{D}^{2}
$$

where:

$$
\|\bullet\|_{\mathcal{S}}^{2}=\int_{\mathbf{H}_{\mathrm{m}} \times \mathbf{H}_{\mathrm{d}}} \int_{\Omega} \operatorname{Tr}\left[\bullet \mathbf{K}^{-1} \bullet\right] \mathrm{d} \Omega \mathrm{d} P(\underline{m}) \mathrm{d} Q(\underline{d})
$$




$$
\|\bullet\|_{\mathcal{U}}^{2}=\int_{\mathbf{H}_{\mathrm{m}} \times \mathbf{H}_{\mathrm{d}}} \int_{\Omega} \operatorname{Tr}[\varepsilon(\bullet) \mathbf{K} \varepsilon(\bullet)] \mathrm{d} \Omega \mathrm{d} P(\underline{m}) \mathrm{d} Q(\underline{d})
$$

\section{Remarks:}

- The constitutive relation error can be easily extended to ill-posed problems where $\partial_{1} \Omega \cap \partial_{2} \Omega \neq\{\oslash\}$.

- The Prager-Synge theorem can also be extended. Furthermore, it is valid, along with the type of result given by Property 2 , for all values of $\underline{m}$ and $\underline{d}$.

- It is possible to introduce "means" and "standard deviations" into the above expressions.

\section{$3 \quad$ Validation of the model}

\subsection{The validation problem in the stochastic framework}

We are seeking to validate the linear elasticity model described in Section 2.1 in the case where $\underline{f}_{\mathrm{d}}=\underline{0}$. This model is probabilistic and defined by:

$$
\mathbf{K}(\underline{m}(\theta))
$$

where $\underline{m}(\theta)$ is a random variable whose probability density function is $\mathrm{d} P(\underline{m})$. The corresponding space is $\mathbf{H}_{\mathrm{m}}$.

The validation is carried out based on experimental results:

- $\underline{\tilde{U}}_{\mathrm{d}}(\underline{\tilde{d}}(\theta))$ over $\partial_{1} \Omega$

- $\underline{\tilde{F}}_{\mathrm{d}}(\underline{\tilde{d}}(\theta))$ over $\partial_{2} \Omega$

where $\tilde{\tilde{d}}(\theta)$ is a random variable whose probability density function is $\mathrm{d} \tilde{Q}(\underline{\tilde{d}})$. The corresponding space is $\mathbf{H}_{\mathrm{d}}$.

All the quantities introduced here are assumed to be perfectly known:

- model: $\mathbf{K}(\underline{m}), \mathrm{d} P(\underline{m}), \mathbf{H}_{\mathrm{m}}$

- experimental results: $\underline{\tilde{U}}_{\mathrm{d}}(\underline{\tilde{d}}(\theta)), \underline{\tilde{F}}_{\mathrm{d}}(\underline{\tilde{d}}(\theta)), \mathrm{d} \tilde{Q}(\underline{\tilde{d}}), \mathbf{H}_{\mathrm{d}}$

\subsection{Validation using the modified-CRE: general philosophy}

The idea introduced in [18] and developed, principally at LMT-Cachan, over the last twenty years consists in using as references: 
- the reliable experimental information (e.g. positions of the sensors, eigenfrequencies)

- part of the model, described by reliable equations (e.g. the equilibrium equations)

Thus, what is considered to be the reference is verified exactly, while the rest, i.e. the constitutive relations and the remaining experimental information, is verified in an average sense. The modified constitutive relation error turns out to be the sum of two errors: the first error is associated with the verification of the constitutive relations, which are a priori unreliable: it is the constitutive relation error, as described above; the second error concerns the unreliable experimental information. This approach is also characterized by the fact that it is the only approach which leads to an actual error and, therefore, which is capable of dealing with the validation problem. For further details in the context of structural dynamics, see $[20,22]$. The latest developments can be found in $[20,39,28]$.

Let us note that the approaches described in $[40,25,26]$ can be viewed as variations of the modified constitutive relation error method. For more details on the validation of deterministic models, see $[5,6,12,9,15]$.

The stochastic case has rarely been considered. A complete parallel with Tarantola's theory [34] was identified recently in [38]. This option requires a choice of norm of the "statistical" type for the unreliable experimental information, a direction which, until now, has been followed only in [37]. A recent work [38] showed that this "statistical" choice is not absolutely required if the experimental data are uncertain. In other words, the norm currently used, which is "mechanical" by nature, is at least equally effective; it filters the experimental noise relatively well.

\subsection{The CRE approach - Deterministic case}

Let $\underline{z}=(\underline{u}, \underline{F})$ representing the boundary conditions for both displacements and forces. The space of the admissible $s=(\underline{U}, \sigma, \underline{z})$, denoted $\mathbf{S}_{\mathrm{ad}}$, is the space of the solutions of:

- the kinematic constraint equations $\underline{U} \in \mathcal{U}$

$$
\underline{U}_{\partial_{1} \Omega}=\underline{u}
$$

- the equilibrium equations $\sigma \in \mathcal{S}$

$$
\begin{gathered}
\underline{\operatorname{div}} \sigma=\underline{0} \\
\sigma \underline{\eta}_{\partial_{2} \Omega}=\underline{F}
\end{gathered}
$$


Here, the intersection of $\partial_{1} \Omega$ and $\partial_{2} \Omega$ is usually nonempty; were it empty, would the problem not be ill-posed anymore, and the solution be easily determined.

Then, the modified constitutive relation error for $s \in \mathbf{S}_{\mathrm{ad}}$ is:

$$
\begin{aligned}
\eta^{2}(s)= & \int_{\Omega} \operatorname{Tr}\left[(\sigma-\mathbf{K} \varepsilon(\underline{U})) \mathbf{K}^{-1}(\sigma-\mathbf{K} \varepsilon(\underline{U}))\right] \frac{\mathrm{d} \Omega}{D^{2}} \\
& +\frac{r}{1-r}\left\{\left\|\underline{u}-\underline{\tilde{U}}_{\mathrm{d}}\right\|_{1}^{2}+\left\|\underline{F}-\underline{\tilde{F}}_{\mathrm{d}}\right\|_{2}^{2}\right\}
\end{aligned}
$$

$r$ is a weighting coefficient representing how much we trust the experimental results. Due to the denominator $D$ and the choice of the norms \|\|$\|$, the two terms of the error have equivalent weights. The norms $\|\mid\| \|_{1}$ and $\|||\|_{2}$ are the classical energy norms which define $\left[\mathbf{H}^{1 / 2}\left(\partial_{1} \Omega\right)\right]^{3}$ and $\left[\mathbf{H}^{-1 / 2}\left(\partial_{2} \Omega\right)\right]^{3}$ : they characterize the unreliable experimental information.

The problem to be solved becomes:

Find $s \in \mathbf{S}_{\mathrm{ad}}$ which minimizes:

$$
\begin{aligned}
s & \rightarrow \eta^{2}(s)=E_{C R E}^{2}(s)+\frac{r}{1-r} I^{2}(s) \\
\mathbf{S}_{\mathrm{ad}} & \rightarrow \mathbb{R}
\end{aligned}
$$

For convenience, this problem can be described in terms of displacements by introducing $\underline{V} \in \mathcal{U}$ such that:

$$
\begin{gathered}
\underline{\operatorname{div}} \mathbf{K} \varepsilon(\underline{V})=\underline{0} \\
\mathbf{K} \varepsilon(\underline{V}) \underline{n_{\partial_{2} \Omega}}=\underline{F}
\end{gathered}
$$

$(\underline{V}-\underline{U})=\underline{0}$ over the complementary part of $\partial_{2} \Omega$

Let $\gamma=(\underline{U}, \underline{V}, \underline{z})$ and let $\Gamma_{\mathrm{ad}}$ be the space of the admissible $\gamma$. The above problem becomes:

Find $\gamma \in \Gamma_{\text {ad }}$ which minimizes:

$$
\begin{aligned}
\gamma & \rightarrow \eta^{2}(\gamma) \\
\Gamma_{\mathrm{ad}} & \rightarrow \mathbb{R}
\end{aligned}
$$

with:

$$
\begin{aligned}
\eta^{2}(\gamma)= & \int_{\Omega} \operatorname{Tr}[(\varepsilon(\underline{U}-\underline{V})) \mathbf{K}(\varepsilon(\underline{U}-\underline{V}))] \frac{\mathrm{d} \Omega}{D^{2}} \\
& +\frac{r}{1-r}\left\{\left\|\underline{u}-\underline{\tilde{U}}_{\mathrm{d}}\right\|_{1}^{2}+\left\|\underline{F}-\underline{\tilde{F}}_{\mathrm{d}}\right\|_{2}^{2}\right\}
\end{aligned}
$$


In order to go further, we will now work only with the boundary quantities $\underline{z}$, and the discretized form:

$$
\begin{array}{|l}
\text { Find } \underline{z} \in \mathbb{R}^{n} \text { which minimizes: } \\
\qquad \begin{aligned}
\underline{z} & \rightarrow \eta^{2}(\underline{z}) \\
\mathbb{R}^{n} & \rightarrow \mathbb{R}
\end{aligned} \\
\text { with } \eta^{2}(\underline{z})=\underline{z}^{T} \mathbb{H} \underline{z}+\frac{r}{1-r}(\underline{z}-\underline{\tilde{z}})^{T} \mathbb{Z}(\underline{z}-\underline{\tilde{z}}) .
\end{array}
$$

One has:

$$
\left[\mathbb{H}+\frac{r}{1-r} \mathbb{Z}\right] \underline{z}=\frac{r}{1-r} \mathbb{Z} \underline{\tilde{z}}
$$

where the operator $\mathbb{H}+\frac{r}{1-r} \mathbb{Z}$ is regular.

Remark: Today, for the weighting coefficient $r$ we systematically use the value 0.5 ; arguments in favor of this value are given later in the paper.

The calculation of the error and the correction procedure can be expensive for industrial structures. To alleviate this problem, ad hoc reduced bases were introduced in [39].

One might question the influence of the finite element discretization on the value of the error and on the updating process. In [41], it was shown that the updating process can counterbalance the discretization errors only in exceptional cases.

\subsection{Extension of the CRE approach - noise-free measurements}

The experimental data $\underline{\tilde{z}}=\left(\underline{\tilde{U}}_{\mathrm{d}}, \underline{\tilde{F}}_{\mathrm{d}}\right)$ are random, but in this section we will assume that they are not affected by measurement errors; their probability density function is $\mathrm{d} \tilde{Q}(\underline{\tilde{z}})$ and the corresponding space is $\mathbf{H}_{\mathbf{z}}$.

\subsection{1 z-value given by the model}

Let $\underline{z}=(\underline{u}, \underline{F})$ calculated by the model. $\left(\underline{u}=\underline{U}_{\mid \partial_{1} \Omega}, \underline{F}=\sigma \underline{\eta}_{\partial_{2} \Omega}\right)$ In discretized form, $\underline{z}$ is characterized by:

$$
\mathbb{H} \underline{z}=\underline{0}
$$

where the operator $\mathbb{H}$ is the one defined in (9) and (10). Next, let us split $\underline{z}$ into two parts $\underline{z}_{I}$ and $\underline{z}_{R}$, where $\underline{z}_{I}$ is the excitation which gives the model a unique response such that:

$$
\underline{z}_{R}=\mathbb{Q}(\underline{m}(\theta)) \underline{z}_{I}
$$


Thus, relation (12) is a modified form of (11). As an illustration, consider the case of a forced-vibration problem discretized with the finite element method: $\underline{z}_{I}$ is the nodal forces vector, whereas $\underline{z}_{R}$ is the nodal response vector; if $\omega$ is the angular frequency of the excitation, $\mathbb{Q}(\underline{m}(\theta))=(\mathbf{K}(\underline{m}(\theta))+\mathrm{i} \omega \mathbf{B}(\underline{m}(\theta))-$ $\left.\omega^{2} \mathbf{M}(\underline{m}(\theta))\right)^{-1}$ with the general case where global stiffness, damping and mass matrices depend on the random variable $\underline{m}(\theta)$.

An important point is that here $\underline{z}_{I}$ is assumed to be perfectly known, in a deterministic sense or not. In order to simplify the presentation, we will first examine the deterministic case, which is also the most usual case in practice. The general case will be considered later.

Here, the difficulty in deriving error estimators for validation purposes resides in the condition that for an "exact" stochastic model the error should be zero.

Definition: A stochastic model is said to be "exact" if for any excitation the probability density function of $\underline{z}$ calculated by the model is equal to the probability density function of $\underline{z}$ derived from the experimental data.

The scientific stumbling block resides, on the one hand, in the description of the experimental values (which, in practice, is very succinct) and, on the other hand, in the comparison of the experimental values with the values given by the model.

The guiding principle in the approach we are proposing to solve this problem consists in observing that there are, in fact, two sources of perturbation [4]:

- one is associated with the model itself (indicator $\mu$ )

- the other is related to the fluctuations inherent in the stochastic nature of the model (indicator $\mu^{\prime}$ )

The first step consists in considering the case where the model is "exact" $(\mu=0)$; the solution calculated with the model can be written:

$$
\underline{z}(\underline{m}(\theta))=\underline{\bar{z}}+\mu^{\prime}\left[\begin{array}{c}
{[\mathbb{Q}-\overline{\mathbb{Q}}] \overline{\bar{z}}_{I}} \\
0
\end{array}\right]
$$

where $\boldsymbol{\bullet}=\int_{\mathbf{H}_{\mathrm{m}}} \bullet \mathrm{d} P(\underline{m})$. This quantity must be compared with the experimental data.

For a nearly similar model $(\mu \neq 0)$, if one neglects the second-order terms, the solution calculated with the model can be written:

$$
\underline{z}(\underline{m}(\theta))+\mu \Delta \underline{z}(\underline{m}(\theta)) \approx \underline{\bar{z}}+\mu \Delta \underline{\bar{z}}+\mu^{\prime}\left[\begin{array}{c}
{[\mathbb{Q}-\overline{\mathbb{Q}}] \underline{\bar{z}}_{I}} \\
0
\end{array}\right]
$$


In other words, one can say that in first-order approximation the errors on the model itself affect the mean value, and nothing else than the mean value.

\subsubsection{Reconstruction of experimental data}

Expression (14) plays a major role in what we call "reconstructed experimental data". Let us recall that from the experimental side very little statistical information is available. Thus, it seems reasonable when the excitation is deterministic and the measurements are noise-free to use only the mean value. Consequently, the reconstructed experimental data is:

$$
\left[\begin{array}{c}
\left\langle\underline{\tilde{z}}_{R}\right\rangle \\
\underline{\tilde{z}}_{I}
\end{array}\right]+\left[\begin{array}{c}
{[\mathbb{Q}(\underline{m}(\theta))-\overline{\mathbb{Q}}] \underline{\tilde{z}}_{I}} \\
0
\end{array}\right]
$$

where $\langle\underline{\tilde{z}}\rangle=\int_{\mathbf{H}_{\mathbf{z}}} \tilde{\tilde{z}} \mathrm{~d} \tilde{Q}(\underline{\tilde{z}})$.

When the excitation $\underline{\tilde{z}}_{I}$ is a known random function, one has:

$$
\underline{\tilde{z}}_{I}(\tilde{\tilde{l}}(\theta))
$$

where $\tilde{l}$ is a vector random variable whose probability density function and associated space are $\mathrm{d} \tilde{L}(\tilde{\tilde{l}})$ and $\mathbf{H}_{1}$ respectively. In the general case, the reconstructed experimental data is:

$$
\left[\begin{array}{c}
\left\langle\underline{\tilde{z}}_{R}\right\rangle \\
\underline{\tilde{z}}_{I}
\end{array}\right]+\left[\begin{array}{c}
\mathbb{Q}(\underline{m}(\theta)) \underline{\tilde{z}}_{I}(\underline{\tilde{l}}(\theta))-\overline{\mathbb{Q}} \int_{\mathbf{H}_{1}} \underline{\tilde{z}}_{I} \mathrm{~d} \tilde{L}(\underline{\tilde{l}}) \\
0
\end{array}\right]
$$

\subsubsection{The constitutive relation error for validation}

Let us proceed with the usual situation where the excitation is deterministic. In this case, the modified constitutive relation error is:

$\eta^{2}(\underline{z})=\int_{\mathbf{H}_{\mathrm{m}}}\left[\underline{z}^{T} \mathbb{H} \underline{z}+\frac{r}{1-r}(\underline{z}-\langle\underline{\tilde{z}}\rangle-(\mathbb{L}-\overline{\mathbb{L}})\langle\underline{\tilde{z}}\rangle)^{T} \mathbb{Z}(\underline{z}-\langle\underline{\tilde{z}}\rangle-(\mathbb{L}-\overline{\mathbb{L}})\langle\underline{\tilde{z}}\rangle)\right] \mathrm{d} P(\underline{m})$

with:

$$
\mathbb{L} \underline{\tilde{z}}=\left[\begin{array}{c}
\mathbb{Q} \underline{\tilde{z}}_{I} \\
0
\end{array}\right]
$$

$\underline{z}$ is a function of $\underline{m}$; let $\mathbf{Z}_{\mathrm{m}}$ be the corresponding space. Then, the problem associated with the error calculation is: 
Find $\underline{z} \in \mathbf{Z}_{\mathrm{m}}$ which minimizes:

$$
\begin{aligned}
\underline{z}^{\prime} & \rightarrow \eta^{2}\left(\underline{z}^{\prime}\right) \\
\mathbf{Z}_{\mathrm{m}} & \rightarrow \mathbb{R}
\end{aligned}
$$

Property 3: If $\mathbb{H}$ is a positive operator and $\mathbb{Z}$ a positive definite operator, the previous problem has only one solution for $0<r<1$.

Proof: It suffices to prove uniqueness. Let $\Delta$ be the difference between two solutions. The minimum conditions yield:

$$
\int_{\mathbf{H}_{\mathrm{m}}}\left[\underline{\Delta}^{T} \mathbb{H} \underline{\Delta}+\frac{r}{1-r} \underline{\Delta}^{T} \mathbb{Z} \underline{\Delta}\right] \mathrm{d} P(\underline{m})=0 \quad(0<r<1)
$$

and, therefore, $\underline{\Delta}=0$, which demonstrates uniqueness.

Fundamental Property 4: With the conditions of Property 3, the modified constitutive relation error is zero if and only if the model is "exact" with respect to the experimental data.

Proof: The error is zero if and only if $\forall \underline{m} \in \mathbf{H}_{\mathrm{m}}$ :

$$
\begin{gathered}
\mathbb{H} \underline{z}=\underline{0} \\
\underline{z}=\langle\underline{\tilde{z}}\rangle+(\mathbb{L}-\overline{\mathbb{L}})\langle\underline{\tilde{z}}\rangle
\end{gathered}
$$

The first condition is equivalent to saying that $\underline{z}$ verifies the equations of the model exactly; then, $\underline{z}$ can be written:

$$
\underline{z}=\left[\begin{array}{c}
\mathbb{Q}(\underline{m}) \underline{z}_{I} \\
\underline{z}_{I}
\end{array}\right]
$$

The second equation can also be written $\forall \underline{m} \in \mathbf{H}_{\mathrm{m}}$ :

$$
\begin{gathered}
\mathbb{Q} \underline{z}_{I}=\left\langle\underline{\tilde{z}}_{R}\right\rangle+[\mathbb{Q}-\overline{\mathbb{Q}}] \underline{\tilde{z}}_{I} \\
\underline{z}_{I}=\underline{\tilde{z}}_{I}
\end{gathered}
$$

and, therefore,

$$
\underline{\bar{z}}=\langle\underline{\tilde{z}}\rangle
$$

Therefore, the reconstructed experimental data is:

$$
\underline{\bar{z}}+(\mathbb{L}(\underline{m})-\overline{\mathbb{L}}) \underline{\bar{z}}
$$

or:

$$
\mathbb{L}(\underline{m}) \underline{\bar{z}}=\underline{z}_{R}
$$




\subsubsection{Example}

Let us consider a beam made up of two homogeneous parts (Young's modulus $E_{0}$ ) connected by a joint (Young's modulus $E_{1}$ ). This beam is subjected to an excitation $F$ at frequency $\omega$, as described in Figure 2. The frequency range being studied comprises the first five modes. Table 1 summarizes the main characteristics of the beam.

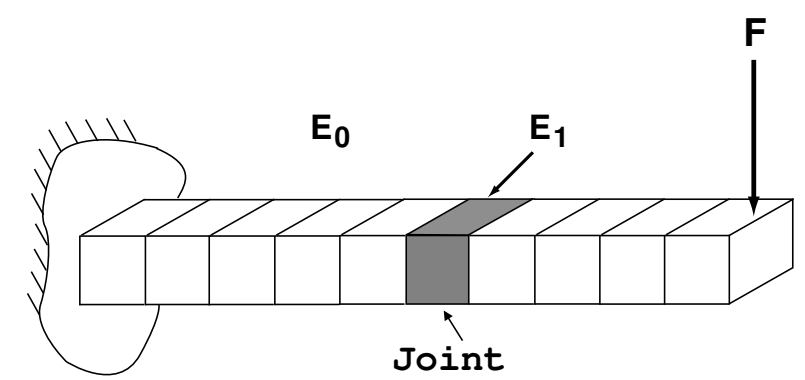

Table 1

Fig. 2. Beam with a joint

Main characteristics

\begin{tabular}{|l|l|}
\hline Length & $10 \mathrm{~m}$ \\
Section & $1.10^{-2} \mathrm{~m}^{2}$ \\
Moment of inertia & $8.33 .10^{-6} \mathrm{~m}^{4}$ \\
Density & $2,700 \mathrm{~kg} / \mathrm{m}^{3}$ \\
\hline
\end{tabular}

What we mean by the "model" is defined in Table 2, where two types of beams are considered. The Young's moduli are modeled in the form:

$$
e=E(1+\alpha \delta)
$$

where $\delta$ is a modified Gaussian variable (such that $e \geq a E, a>0$ ) with zero mean and unit standard deviation.

For this academic example, the measurements were simulated by introducing a hysteretic damping factor of $1 \%$ and considering a frequency range containing the first five modes. The values retained were those which would have been given by ten equally-spaced accelerometers along the beam. Table 3 gives the values of the parameters $E, \alpha$ used to derive these experimental values. Here, we did not introduce any measurement noise.

Table 2

Stochastic parameters for the model (two cases)

\begin{tabular}{|c|cc|}
\hline & $E_{0}, \alpha_{0}$ & $E_{1}, \alpha_{1}$ \\
\hline Case 1 & $71.10^{9} \mathrm{~Pa}, 0$ & $71.10^{9} \mathrm{~Pa}, 0.1$ \\
\hline Case 2 & $71.10^{9} \mathrm{~Pa}, 0.02$ & $71.10^{9} \mathrm{~Pa}, 0.02$ \\
\hline
\end{tabular}


Table 3

Stochastic parameters used to define the measurements

\begin{tabular}{|c|cc|}
\hline & $E_{0}, \alpha_{0}$ & $E_{1}, \alpha_{1}$ \\
\hline Case 1 & $71.10^{9} \mathrm{~Pa}, 0$ & $\mathbf{1 4 2 . 1 0} \mathbf{9}^{\mathbf{P}} \mathrm{Pa}, 0.1$ \\
\hline Case 2 & $71.10^{9} \mathrm{~Pa}, \mathbf{0 . 0 5}$ & $71.10^{9} \mathrm{~Pa}, \mathbf{0 . 0 5}$ \\
\hline
\end{tabular}

For both cases, the spatial distribution of the constitutive relation error was calculated (Figure 3). For Case 1, there is a peak in the joint, which is consistent with the fact that this was the most poorly modeled element. The error distribution for Case 2 is also consistent with a uniform modeling error along the beam. Figure 3 also gives the constitutive relation error, which is an indicator of the quality of the model, and the error in the experimental results.

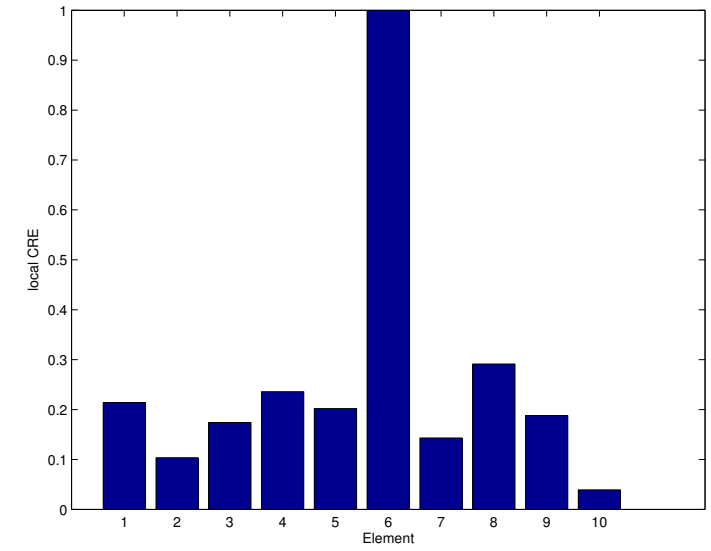

$\bar{E}_{C R E}^{2}(s)=6.8 \%, \bar{I}^{2}(s)=5.6 \%$

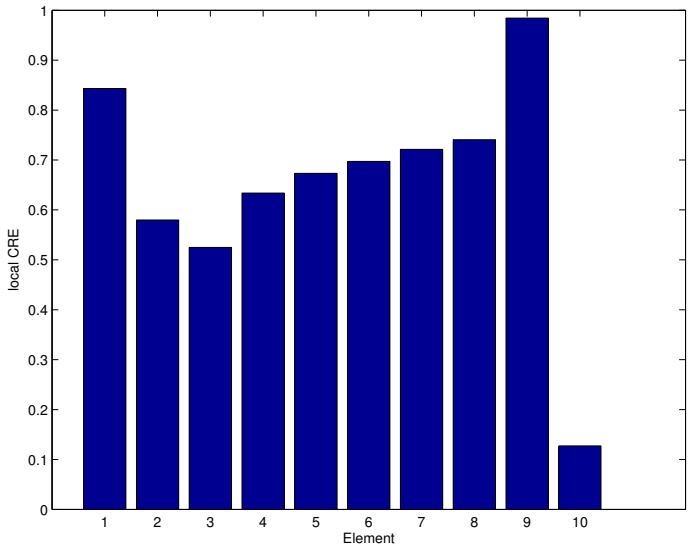

$\bar{E}_{C R E}^{2}(s)=2.0 \%, \bar{I}^{2}(s)=0.2 \%$

Fig. 3. Error values for the two cases

\subsection{Extension of the CRE-approach-measurements with noise}

The model is probabilistic and the experimental data are affected by measurement noise. More precisely, the fluctuations $\underline{\tilde{\delta}}$ due to the measurement noise are defined by the probability density function $\mathrm{d} \tilde{Q}(\underline{\tilde{\delta}})$. The associated Hilbert space is $\mathbf{H}_{\delta}$. If the measurement noise is Gaussian, the probability density function is defined by the covariance matrix, which can reasonably be considered to be diagonal, with a generic term $\sigma^{2}$. Of course, one can easily introduce the value of the measured quantity into the expression of the standard deviation. The important point is that the measurement noise be a fully-described random phenomenon.

In order to simplify the presentation, the excitation will be assumed to be 
deterministic, but, of course, affected by measurement noise. Then, the constitutive relation error is:

$$
\eta^{2}(\underline{z})=\int_{\mathbf{H}_{\mathrm{m}} \times \mathbf{H}_{\delta}}\left[\underline{z}^{T} \mathbb{H} \underline{z}+\frac{r}{1-r}(\underline{z}-\underline{\tilde{z}})^{T} \mathbb{Z}(\underline{z}-\underline{\tilde{z}})\right] \mathrm{d} P(\underline{m}) \mathrm{d} \tilde{Q}(\underline{\tilde{\delta}})
$$

with

$$
\underline{\tilde{z}}(\underline{\tilde{\delta}})=\langle\underline{\tilde{z}}\rangle+\underline{\tilde{\delta}}+(\mathbb{L}-\overline{\mathbb{L}})[\langle\underline{\tilde{z}}\rangle+\underline{\tilde{\delta}}]
$$

where $\langle\underline{\tilde{z}}\rangle$ is the statistical mean of $\underline{\tilde{z}}$. In addition, we consider a noise such that:

Let us recall that:

$$
\int_{\mathbf{H}_{\delta}} \underline{\tilde{\delta}} \mathrm{d} \tilde{Q}(\underline{\tilde{\delta}})=\underline{0}
$$

$\bullet \boldsymbol{\bullet}=\int_{\mathbf{H}_{\mathrm{m}}} \bullet \mathrm{d} P(\underline{m})$

- $\mathbb{L}(\underline{m})=\left[\begin{array}{cc}0 & \mathbb{Q}(\underline{m}) \\ 0 & 0\end{array}\right]$

The associated problem is:

Find $\underline{z}(\underline{m}, \underline{\tilde{\delta}})$ which minimizes

$$
\begin{aligned}
\underline{z}^{\prime} & \rightarrow \eta^{2}\left(\underline{z}^{\prime}\right) \\
\mathbf{Z}_{m, \delta} & \rightarrow \mathbb{R}
\end{aligned}
$$

This problem leads to the resolution of $\forall(\underline{m}, \underline{\tilde{\delta}}) \in \mathbf{H}_{\mathrm{m}} \times \mathbf{H}_{\delta}$ :

$$
\left[\mathbb{H}(\underline{m})+\frac{r}{1-r} \mathbb{Z}(\underline{m})\right] \underline{z}=\frac{r}{1-r}[\mathbb{I}+(\mathbb{L}(\underline{m})-\overline{\mathbb{L}})](\langle\underline{\tilde{z}}\rangle+\underline{\tilde{\delta}})
$$

which can be solved using the polynomial chaos technique. Here, the error is never zero; it is polluted by the measurement noise.

Remark: The validation method for a stochastic model in an uncertain environment which we just developed can be transposed with no major difficulty to time-dependent nonlinear problems, either in quasi-static or in dynamic situations. However, the minimization of the modified constitutive relation error may lead to a problem whose resolution using the polynomial chaos technique is very computationally intensive. The question of the improvement of this resolution should probably be addressed.

\section{Application to an industrial structure}

Before illustrating the extension of the constitutive relation error to the stochastic case, let us briefly review this approach in the deterministic case. Its exten- 
sion to the stochastic case will not be detailed here; it follows the same steps we developed in Section 3.

\subsection{Validation of dynamics models: review of the deterministic case}

We are assuming small displacement and we seek harmonic solutions at the angular frequency $\omega$, with the following equilibrium equation:

$$
\underline{\operatorname{div}} \sigma+\underline{f} \underline{d}(\underline{d}(\theta))=\underline{\Gamma}
$$

with:

where $\rho$ is the density.

$$
\underline{\Gamma}=\rho \frac{\partial^{2} \underline{U}}{\partial t^{2}}
$$

We need some data in addition to the equations presented above (frequency, direction and amplitude of the excitation, boundary conditions, ...) in order to solve the problem. In the framework of model updating and validation, these data come from measurements on real structures. For example, let us consider a structure excited at certain points and whose displacements are measured at other locations. In this example, we partition the data as follows:

\section{The reliable measurements:}

- the measured angular frequency $\omega$;

- the positions and directions of the excitation and sensors.

\section{The less reliable measurements:}

- the amplitudes of the forces ${\underline{F_{\mathrm{d}}}}_{\text {and displacements }} \underline{\tilde{U}}_{\mathrm{d}}$ at the points of excitation;

- the amplitudes of the displacements at the sensor points $\underline{\tilde{U}}_{\omega}$, which constitute a finite-dimension vector containing all the discrete measurement values.

This partitioning is given only as an example and can vary depending on the problem. The reliable quantities and equations define the admissible solution. We seek a solution which is admissible and which verifies the less reliable equations and quantities as perfectly as possible. The problem we need to solve is:

Find $s \in \mathbf{S}_{a d}^{\omega}$

which minimizes $\eta_{\omega}^{2}\left(s^{\prime}\right)$ with $s^{\prime} \in \mathbf{S}_{\text {ad }}^{\omega}$

where $\eta_{\omega}^{2}\left(s^{\prime}\right)$ is the modified constitutive relation error. In the case of a single excitation, the measured displacements are normalized by the amplitude of 
the force vector; thus, only the amplitudes of the displacements appear in the expression of the modified error, which can be written at a given frequency as:

$$
\eta_{\omega}^{2}(s)=\frac{E_{C R E_{\omega}}^{2}(s)}{D_{\omega}^{2}}+\frac{r}{1-r} \frac{\left\|\Pi \underline{U}-\underline{\tilde{U}}_{\omega}\right\|^{2}}{\left\|\underline{\tilde{U}}_{\omega}\right\|^{2}}
$$

where $E_{C R E_{\omega}}^{2}(s)$ is the constitutive relation error and $D_{\omega}^{2}$ represents the energy of the structure at the frequency $\omega$; therefore the first term is dimensionless. The second term represents the error in the measurements and is also dimensionless. For simplicity, we will write:

$$
\eta_{\omega}^{2}(s)=E_{C R E_{\omega r}^{2}}^{2}(s)+\frac{r}{1-r} I_{\omega r}^{2}(s)
$$

where:

$$
\begin{gathered}
E_{C R E_{\omega r}}^{2}(s)=\frac{E_{C R E_{\omega}^{2}}(s)}{D_{\omega}^{2}} \\
I_{\omega r}^{2}(s)=\frac{\left\|\Pi \underline{U}-\underline{\tilde{U}}_{\omega}\right\|^{2}}{\left\|\underline{\tilde{U}}_{\omega}\right\|^{2}}
\end{gathered}
$$

The CRE is greater than or equal to zero. It is equal to zero if the solution $s$ verifies the constitutive relations. $\eta_{\omega}^{2}(s)$ contains all the less reliable quantities and equations which are to be verified by the admissible solution as closely as possible.

$\Pi$ is a projection operator which, when applied to vector $\underline{U}$, yields the value of the vector at the sensors. $\|\bullet\|^{2}$ is a norm. The coefficient $\frac{r}{1-r}$ is a weighting factor which enables us to assign a greater or lesser degree of confidence to the measurements. The optimum choice for $r$ will be discussed later.

In the case of multiple excitations, additional terms appear in the expression of $\eta_{\omega}^{2}(s)$ in order to reflect the fact that the amplitudes of the measured forces are unreliable quantities. For more details, see [22].

Remark: There are other examples where one could consider that the direction or the position of the excitation is unreliable. In these cases, the definitions of the modified constitutive relation error and of admissibility should be changed to reflect this new assumption.

The second term of the modified CRE can be expressed as:

$$
I_{\omega r}^{2}=\frac{\left\|\Pi \underline{U}-\tilde{U}_{\omega}\right\|^{2}}{\left\|\tilde{U}_{\omega}\right\|^{2}}
$$


This term can be normalized and divided into contributions from each sensor at frequency $\omega$ :

$$
I_{\omega r}^{2}=\sum_{i=1}^{N S} \frac{\left\|\Pi \underline{U}-\tilde{U}_{\omega}\right\|_{i}^{2}}{\left\|\tilde{U}_{\omega}\right\|^{2}}=\sum_{i=1}^{N S} I_{i \omega}^{2}
$$

$\|\bullet\|_{i}^{2}$ is the norm obtained by setting all the components of the vector, except that related to sensor $i$, to zero. $N S$ is the number of sensors.

When the structure is studied in a frequency range, we introduce a weighting factor $z(\omega)$ such that:

$$
\int_{\omega_{\min }}^{\omega_{\max }} z(\omega) \mathrm{d} \omega=1 \quad z(\omega) \geq 0
$$

Examples of such weighting factors are:

$$
\begin{aligned}
& z(\omega)=\frac{1}{\omega_{\max }-\omega_{\min }} \\
& z(\omega)=\frac{1}{m} \sum_{i=1}^{m} \delta_{\omega_{i}}(\omega)
\end{aligned}
$$

$\delta_{\omega_{i}}$ is the Dirac distribution associated with the value $\omega_{i}$, where $\omega_{i} i \in 1, \ldots m$ denote the free-vibration eigenfrequencies which are comprised between $\omega_{\min }$ and $\omega_{\max }$.

The modeling error in a frequency range is given by:

$$
E_{C R E_{T}^{2}}^{2}=\int_{\omega_{\min }}^{\omega_{\max }} E_{C R E_{\omega r}^{2}}^{2} z(\omega) \mathrm{d} \omega
$$

and the local contributions become:

$$
E_{C R E T, E}^{2}=\int_{\omega_{\min }}^{\omega_{\max }} E_{C R E E \omega}^{2} z(\omega) \mathrm{d} \omega
$$

The error in the measurements is given by:

$$
I_{T}^{2}=\int_{\omega_{\min }}^{\omega_{\max }} I_{\omega r}^{2} z(\omega) \mathrm{d} \omega
$$

and the local contributions become:

$$
I_{i T}^{2}=\int_{\omega_{\min }}^{\omega_{\max }} I_{i \omega}^{2} z(\omega) \mathrm{d} \omega
$$

Now, the modified constitutive relation error is:

$$
\eta_{T}^{2}=E_{C R E_{T}^{2}}^{2}+I_{T}^{2}
$$


The value of $E_{C R E_{T}^{2}}^{2}$ represents the relative quality (in \%) of the numerical model with respect to the measurements.

\subsection{Localization and correction of errors: review of the deterministic case}

This approach is based on the same principles as adaptive mesh refinement, in which each iteration consists in locating the most erroneous regions and correcting these regions. Here, the corrections do not concern mesh parameters, but physical parameters of the mathematical model.

\subsubsection{Preliminary stage: reconstruction of experimental data}

Once the erroneous measurements have been localized, it is possible to remove these data from the measurements or to correct them (correcting this error causes the value of $I_{i T}^{2}$ to decrease at the corresponding sensor). A correction is possible only if the sources of error can be identified from the test reports or simply by trying the most common causes of error, such as errors in the directions of sensors and exciters, gain errors, ...

Once the experimental data have been corrected, the error should be divided almost equally among all the sensors, which means that all the measurements are of equivalent quality.

\subsubsection{Localization of modeling errors}

Now that the measurement values have been updated as correctly as possible, one can update the model based on the updated experimental results. The value of $E_{C R E_{T}^{2}}$ represents the relative quality (in \%) of the numerical model with respect to the measurements in a certain frequency range. Since this value represents an error measure of the model with respect to the measurements considered as the reference, it can be used to decide whether model updating is necessary or not.

If model updating is considered necessary, one starts from our mathematical model, which depends on a number of uncertain parameters (such as the Young's modulus, the thickness or the damping factors of certain parts). One arranges these structural parameters into a vector $\underline{k}$ and calls the corresponding space k. The selection of the "most erroneous" substructures is based on the criterion:

$$
E_{C R E_{T, E}^{2}} \geq \delta \max _{E \in \mathbf{E}} E_{C R E_{E T}^{2}}
$$


with, for example, $\delta=0.8$. Let $\mathbf{Z}$ be the complete set of the substructures which verify (41).

\subsubsection{Correction of modeling errors}

The localization step enables one to select the regions of the structure where the modeling error is large. Only parameters belonging to these substructures are selected for correction. The problem is:

Find $\underline{k} \in \mathbf{k}_{\mathbf{z}}$ which minimizes:

$$
\begin{aligned}
\underline{k} & \longrightarrow J(\underline{k}) \\
\mathbf{k}_{\mathbf{z}} & \longrightarrow \mathbb{R}
\end{aligned}
$$

The functional $J(\underline{k})$ is defined by:

$$
J(\underline{k})=\int_{\omega_{\min }}^{\omega_{\max }} \eta_{\omega}^{2} z(\omega) \mathrm{d} \omega
$$

This is a nonlinear problem with respect to the parameters in $\underline{k}$.

\subsubsection{Interruption of the model updating process}

Once the correction has been made, the value of $E_{C R E_{T}}^{2}$ is recalculated. If it falls below the required quality level $E_{C R E_{0}}^{2}$, the updating process is terminated. If not, a new iteration consisting of a localization step and a correction step is performed. In each iteration, new erroneous substructures may appear as the result of the substructures from the previous stages being corrected. This approach introduces a regularization mechanism into the inverse (illposed) problem. The verification of $E_{C R E_{T}^{2}}^{2}<E_{C R E_{0}}^{2}$ enables one to determine whether the model is valid. There could be situations in which one cannot reduce the error below the required quality level by acting on the model's parameters. This would indicate that errors are present in the model itself and not only in its parameters.

\subsection{Validation and updating of a dynamic model of SYLDA5 - deterministic case}

The structure is a satellite support called SYLDA5 (Figure 4). The purpose of this component is to allow the Ariane 5 launcher to place two satellites into orbit. This structure is made of very different materials in terms of stiffness as well as density, and it includes different types of joints. The geometry is 
axisymmetric and the structure is 10 meters in total height and 5 meters in diameter. The experimental modes were obtained by a force appropriation method using 5 exciters. A total of 260 accelerometers where placed in various parts of the structure.

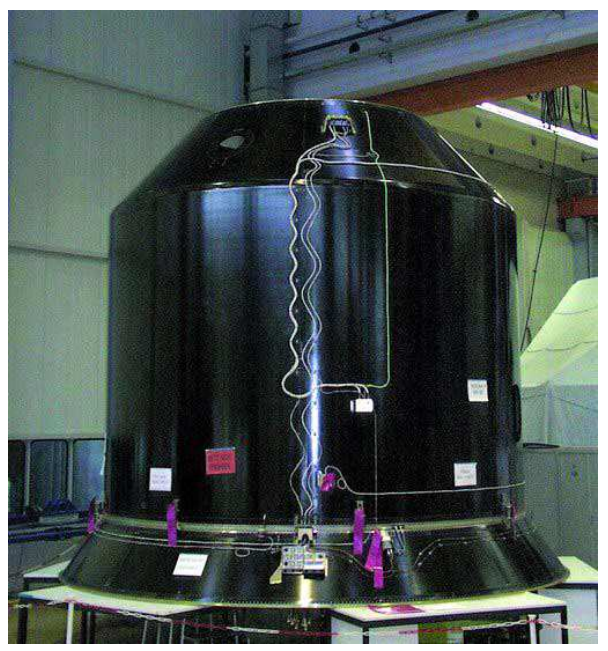

Fig. 4. The SYLDA5 satellite support

The model consists of plate elements for the cylinders and cone and beam elements for the joints and the upper beam of the payload. The first tests have shown that it was essential to take the ground into account in the model; this was done using 3 rotational springs, one translation spring and a rigidbody-movement constraint for all the bottom nodes. The size of the finite element model was decreased from 28,000 to 300 DOFs by using a reduced basis [39]. The model was updated in a previous work [28]. A summary is given in Table 4 . The initial error of $12.39 \%$ was reduced to $2.27 \%$ after four iterations.

Table 4

Summary of the updating process. $\left(^{+}\right.$: mode swapped with Modes 4 and 5)

\begin{tabular}{|c|c|c|c|c|c|c|c|c|c|c|c|}
\hline Iterations & \multicolumn{3}{|c|}{ Initial } & \multicolumn{2}{|c|}{1} & \multicolumn{2}{|c|}{2} & \multicolumn{2}{|c|}{3} & \multicolumn{2}{|c|}{4} \\
\hline Error (\%) & $E_{C R E}$ & $\Delta f$ & MAC & $E_{C R E}$ & $\Delta f$ & $E_{C R E}$ & $\Delta f$ & $E_{C R E}$ & $\Delta f$ & $E_{C R E}$ & $\Delta f$ \\
\hline Mode 1 & 3.45 & -3.51 & 99.62 & 2.65 & -2.69 & 0.24 & 0.28 & 2.05 & 2.05 & 1.67 & 1.86 \\
Mode 2 & 3.25 & -3.31 & 99.73 & 2.46 & -2.49 & 0.39 & 0.37 & 2.19 & 2.15 & 1.54 & 1.43 \\
Mode 3 & 5.10 & -5.24 & 96.53 & 4.06 & -4.14 & 0.40 & -0.40 & 1.49 & 1.48 & 1.49 & 1.48 \\
Mode 4 & 10.29 & -10.85 & 99.11 & 9.78 & -10.29 & 9.03 & -9.48 & 4.09 & -4.15 & 4.28 & -4.22 \\
Mode 5 & 9.86 & -10.39 & 99.64 & 9.36 & -9.83 & 8.61 & -8.99 & 3.66 & -3.75 & 3.76 & -3.98 \\
Mode 6 & 3.47 & $3.45+$ & 97.45 & 1.91 & $1.90+$ & 0.10 & $0.10^{+}$ & 2.66 & 2.63 & 0.00 & 0.00 \\
Mode 7 & 27.15 & 23.60 & 98.77 & 1.27 & 1.27 & 1.27 & 1.27 & 3.36 & 3.30 & 2.71 & 2.67 \\
Mode 8 & 27.77 & 24.09 & 98.87 & 1.92 & 1.90 & 1.92 & 1.90 & 4.00 & 3.92 & 3.36 & 3.30 \\
Mode 9 & 1.78 & -1.79 & 96.14 & 0.09 & -0.09 & 0.09 & -0.09 & 0.02 & 0.02 & 0.10 & -0.10 \\
Mode 10 & 1.24 & -1.24 & 95.84 & 0.44 & 0.44 & 0.44 & 0.44 & 0.55 & 0.55 & 0.43 & 0.43 \\
Mode 11 & 6.41 & $*$ & $*$ & 1.01 & $*$ & 1.04 & $*$ & 2.11 & $*$ & 1.65 & $*$ \\
Mode 12 & 4.82 & $*$ & $*$ & 0.64 & $*$ & 0.63 & $*$ & 1.36 & $*$ & 0.56 & $*$ \\
\hline$E_{C R E_{T}}$ ) & 12.39 & & & 4.32 & & 3.69 & & 2.62 & & 2.27 & \\
\hline
\end{tabular}

Now, let us introduce some randomness into this engineering structure in order to see how the extension of the CRE works in the proposed stochastic case. 


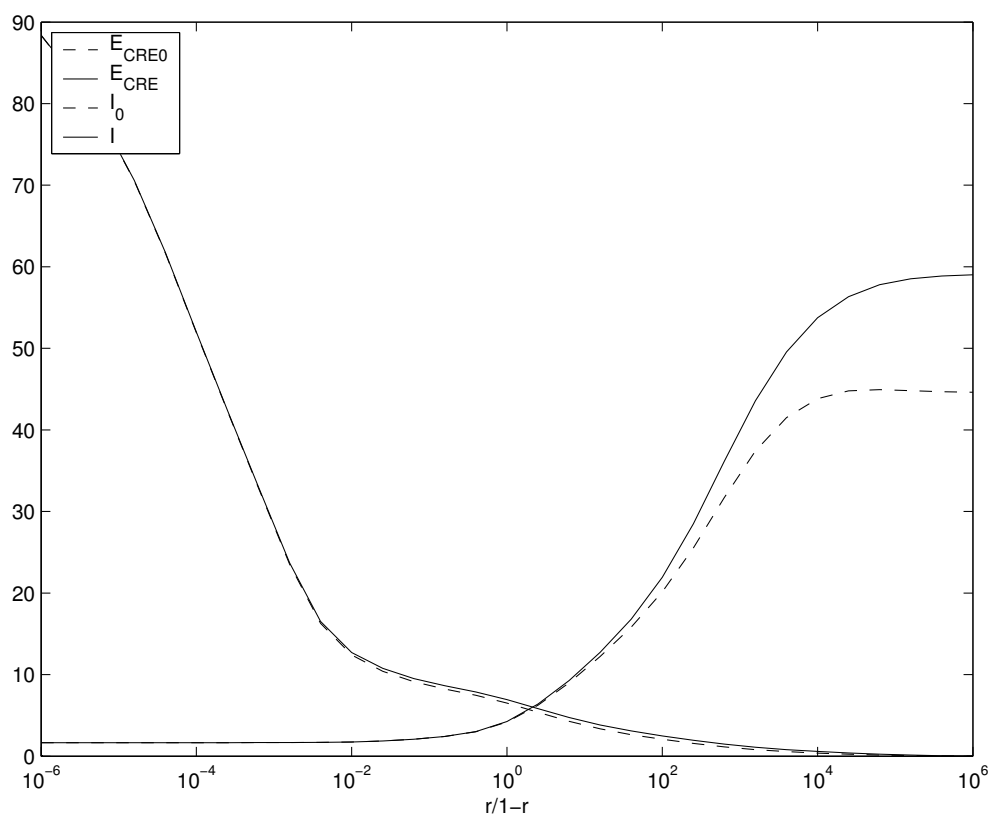

Fig. 5. Errors vs. the weighting factor $\frac{r}{1-r}$

4.4 Validation and updating of SYLDA5 - measurements with noise and deterministic dynamic modeling

We added a $5 \%$ uniform random noise to the measured values. We assumed the mean values to be known.

For the updated model, we calculated the modified constitutive relation error with the two contributions $E_{C R E}$ and $I$.

The values corresponding to the case of noise-free measurements are denoted $E_{C R E 0}$ and $I_{0}$.

Figure 5 gives the values of these errors in terms of the weighting factor $\frac{r}{1-r}$. It is interesting to note that for values close to 1 the effect of noise is very small. Moreover, the curve which gives the model's constitutive relation error $\bar{E}_{C R E}$ in terms of $\frac{r}{1-r}$ is relatively flat, which means that $\bar{E}_{C R E}$ depends very little on $\frac{r}{1-r}$. This is the reason why, at the present time, we systematically take $r=0.5$ for structures similar to SYLDA5.

Figure 6 gives the contributions of the elements to the modified constitutive relation error for the two cases (without and with noise). Here, the value of $\frac{r}{1-r}$ is set relatively high in order to emphasize the influence of the noise. The noise does not appear to modify the localization map very much. 

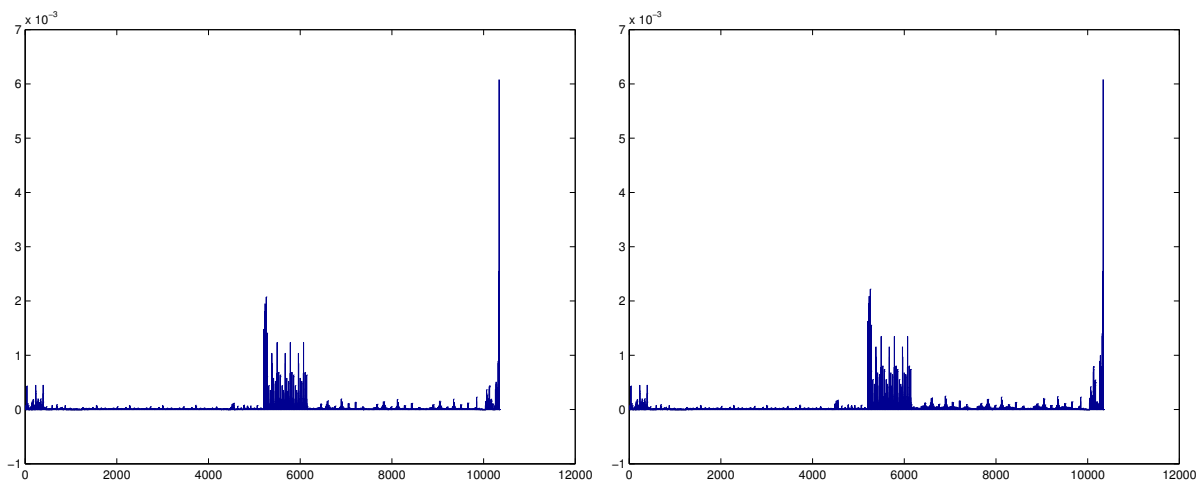

Fig. 6. Element contributions to the relative error, without noise (left) and with noise (right) $\left(\frac{r}{1-r}=10^{4}\right)$

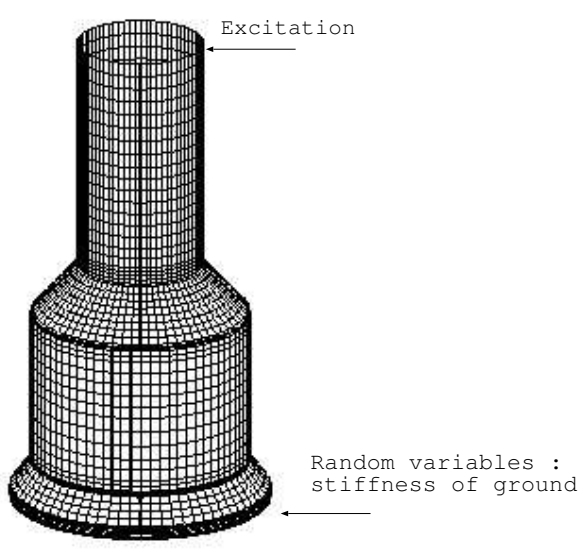

Fig. 7. The structure being studied

\subsection{Validation of SYLDA5 with a probabilistic dynamic model}

This is an academic example in which the experimental data resulted from a calculation. The frequency range being studied contains the first six modes of the structure. A deterministic excitation was applied at the top (see Figure 7). The only random variable was the stiffness of the ground. The Young's modulus was expressed as:

$$
E=E_{0}(1+\alpha \delta)
$$

where $\delta$ is a modified Gaussian variable with zero mean and unit standard deviation.

The "real" model used for the determination of the experimental data was defined by:

$$
E_{0}=3 \quad \alpha=1
$$



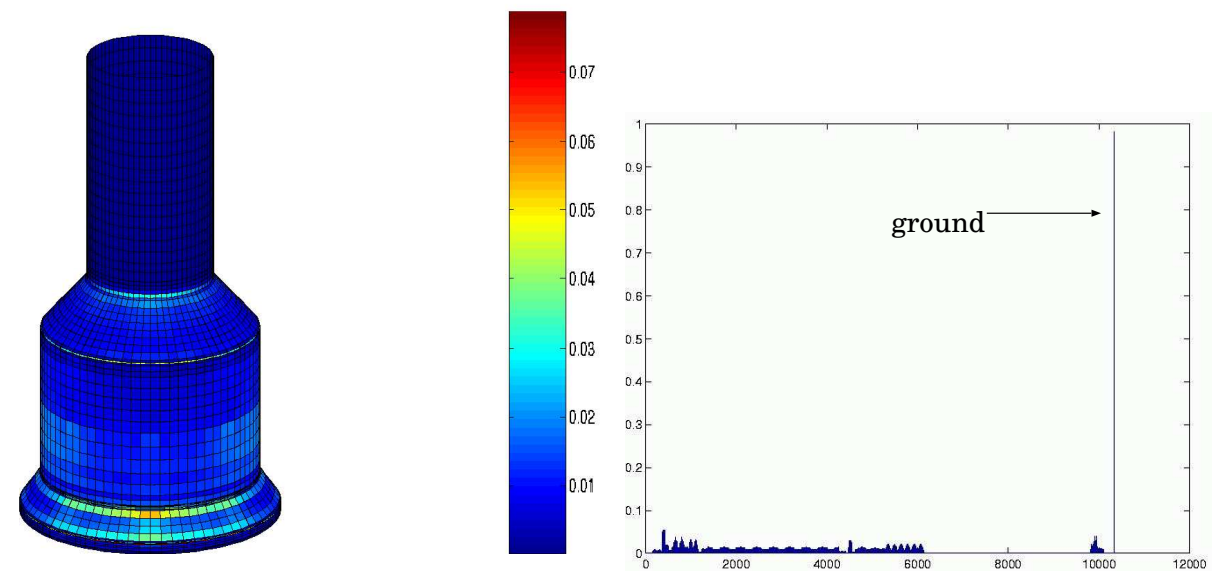

Fig. 8. Error values for the probabilistic dynamic model

We used the same sensors as in Section 4.3.

The model we want to validate is defined by:

$$
E_{0}=1 \quad \alpha=0.5
$$

Figure 8 shows the associated values of the constitutive relation error; we get approximatively the same values, excepted for the stiffness of the ground, whose contribution is much higher. So it is worth noting the accuracy with which the modeling error was localized, which shows the efficiency of the stochastic validation method applied to an industrial-sized problem.

\section{Conclusion}

The validation method we introduced here is very general; it is applicable to probabilistic models in an uncertain experimental environment. The domains of application concern, above all, complex structures which have been finely modeled, for which the model's validation must rely on only scarce experimental data. One major characteristic of this approach, which is definitely "mechanics-oriented", is that it leads to a true error measure which enables one to evaluate the quality of the model. This error measure is the keystone of an updating method which, here, is identical to that which was developed for the deterministic case. This updating method contains a regularization procedure which is inherently non-artificial. This approach, which is an extension of works done in the deterministic framework, follows the guiding principle that part of the model is used as the reference. After updating, there remains a residual error which characterizes a certain lack of knowledge of the model whose evaluation is the focus of a new theory which is under development [42]. 


\section{References}

[1] P. Ladevèze, Advances in Adaptative Computational Methods in Mechanics, P. Ladevèze and J. T. Oden Edition, Elsevier, 1998, Ch. A Modelling Error Estimator for Dynamic Structural Model Updating, pp. 135-154.

[2] P. Ladevèze, J.-P. Pelle, Mastering Calculations in Linear and Nonlinear Mechanics, Springer, (to appear 2004).

[3] I. Babuska, T. Strouboulis, The Finite Element Method and its Reliability, Oxford Science, 2001.

[4] P. Ladevèze, Validation and verification of stochastic models in uncertain environment through the constitutive relation error method, Internal Report 258, LMT-Cachan, in French (June 2003).

[5] J. Mottershead, M. Friswell, Model updating in structural dynamics: a survey, Journal of Sound and Vibration 167 (2) (1993) 347-375.

[6] S. R. Ibrahim, Analytical dynamic model updating: The challenge for the nineties, Transaction of the Institution of Engineers Australia 16 (1) (1991) 17.

[7] M. Baruch, Optimal Correction of Mass and Stiffness Matrices Using Measured Modes, AIAA Journal 20 (11) (1982) 1623-1626.

[8] A. Berman, E. J. Nagy, Improvement of a Large Analytical Model Using Test Data, AIAA Journal 21 (8) (1983) 1168-1173.

[9] M. Kaouk, D. Zimmerman, Structural Damage Assessment Using a Generalized Minimum Rank Perturbation, AIAA Journal 32 (4) (1994) 836-842.

[10] D. Zimmerman, M. Kaouk, Eingenstructure Assignment Approach for Structural Damage Detection, AIAA Journal 30 (7) (1992) 1848-1855.

[11] H. Berger, R. Ohayon, L. Quetin, L. Barthe, P. Ladèveze, M. Reynier, Updating methods for structural dynamics models, La Recherche Aérospatiale 5 (1991) 9-20, in French.

[12] C. Farhat, F. Hemez, Updating finite element dynamic models using an elementby-element sensitivity methodology, AIAA Journal 31 (9) (1993) 1702-1711.

[13] J. Piranda, G. Lallement, S. Cogan, Parametric correction of finite element models by minimization of an output residual: Improvement of the sensivity method, in: Proc. IMAC IX, Firenze, Italy, 1991, pp. 363-368.

[14] S. Lammens, M. Brughmans, J. Leuridan, W. Heylen, P. Sas, Application of a FRF based model updating technique for the validation of a finite element models of components of the automotive industry, in: Proc. Design Engineering Technical Conferences, A.S.M.E. Conferences, Boston, 1995, pp. 1191-1200. 
[15] F. Hemez, S. Doebling, Test-analysis correlation and finite element model updating for nonlinear, transient dynamics, in: Proc. IMAC XVIII, Kissimmee, Florida, 1999, pp. 1501-1510.

[16] S. Doebling, F. Hemez, J. Schulze, A. Cundy, A metamodel-based approach to model validation of nonlinear finite element simulations, in: Proc. IMAC XX, Los Angeles, California, 2002.

[17] R. Shinn, F. Hemez, S. Doebling, Estimating the error in simulation prediction over the design space, in: 44th AIAA/ASME/ASCE/AHS Structures, Structural Dynamics and Materials Conference, Norfolk, Virginia, 2003.

[18] P. Ladevèze, Updating of complex structures models, Tech. Rep. 33.11.01.4, Aérospatiale, Les Mureaux, in French (1983).

[19] P. Ladevèze, M. Reynier, Vibrations Analysis Techniques and Applications, ASME Publishers, 1989, Ch. FE Modeling and Analysis: a Localization Method of Stiffness Errors for the Adjustement of FE Models, pp. 355-361.

[20] P. Ladevèze, Error on the constitutive relation in dynamics : theory and application to model updating in structural dynamics, Internal Report 150, LMT-Cachan, in French (1993).

[21] A. Chouaki, P. Ladevèze, L. Proslier, Updating Structural Dynamic Models with Emphasis on the Damping Properties, AIAA Journal 36 (6) (1998) 10941099.

[22] P. Ladevèze, A. Chouaki, Application of a posteriori error estimation for structural model updating, Inverse Problems 15 (1999) 49-58.

[23] P. Ladevèze, Nonlinear Computational Structural Mechanics, Springer, New York, 1999.

[24] A. Chouaki, A. Deraemaeker, P. Ladevèze, S. Le Loch, Model updating using the error in the constitutive relation : Real case study, in: Proc. IMAC XVIII, San Antonio, Texas, 2000, pp. 140-146.

[25] E. Balmès, Review and evaluation of shape expansion methods, in: Proc. IMAC XVIII, San Antonio, Texas, 2000.

[26] P. Moine, L. Billet, D. Aubry, Updating a non conservative finite element model: two modal methods, in: Proc. Design Engineering Technical Conference Nb. 3, Boston, USA, 1995, pp. 1302-1310.

[27] R. Pascual, J. Golinval, M. Razeto, On the reliability of error localization indicators, in: Proc. ISMA 23, Leuven, Belgium, 1998.

[28] D. Barthe, A. Deraemaeker, P. Ladevèze, S. L. Loch, Validation and updating of models of industrial structures based on the constitutive relation error, AIAA Journal (to appear 2003).

[29] R. Ghanem, P. Spanos, Stochastic Finite Elements : A spectral approach, Springer, Berlin, 1991. 
[30] A. Keese, H. Matthies, Numerical methods and smolyak quadrature for nonlinear stochastic partial differential equations, SIAM J. Sci. Comp. .

[31] G. Schueller, A state-of-the-art report on computational stochastic mechanics, Probabilistic Engineering Mechanics 12 (4) (1997) 197-321.

[32] R. Ghanem, M. Pelissetti, A method for the validation of predictive computations using a stochastic approach, in: Proc. Of OMAE'02, Oslo, Norway, 2002.

[33] R. Ghanem, M. Pelissetti, Error estimation for the validation of model-based predictions, in: Proc. Of 5th World Congress on Computational Mechanics, Vienna, Austria, 2002.

[34] A. Tarantola, Inverse Problem Theory, Elsevier, 1987.

[35] W. Menke, Geophysical data analysis : discrete inverse theory, Academic Press, 1984.

[36] M. Bonnet, J. B. Abdallah, Structural Parameter identification using non linear Gaussian inversion, 1st Edition, Inverse Problems in Engineering, H.D. Bui M. Tanaka et al., Balkema, Rotterdam, 1994, pp. 235-242.

[37] A. Chouaki, Updating of structural dynamical models with damping, Ph.D. thesis, LMT-Cachan, ENS Cachan - Paris 6 University (1997).

[38] A. Deraemaeker, P. Ladevèze, T. Romeuf, Model validation in the presence of uncertain experimental data, Eng. Comp. (to appear 2004).

[39] A. Deraemaeker, P. Ladevèze, P. Leconte, Reduced bases for model updating in structural dynamics based on Constitutive Relation Error, Comp. Meth. in Applied Mech. and Engrg. 191(21-22) (2002) 2427-2444.

[40] J. C. Golinval, P. Collignon, Comparison of model updating methods adapted to local error detection, in: Proc. ISMA 21, Leuven, Belgium, 1996.

[41] A. Deraemaeker, P. Ladevèze, Effect of the finite element discretisation on the dynamic model updating process, in: Proc. IMAC XIX, Kissimmee, Florida, 2001.

[42] P. Ladevèze, G. Puel, T. Romeuf, On a strategy reduction of the lack of knowledge (LOK) of a structural dynamics model, in: Proc. IMAC XXII, Dearborn, Michigan, 2004. 